\title{
Correspondence
}

\section{Texture Classification by Wavelet Packet Signatures}

Andrew Laine and Jian Fan

\begin{abstract}
This correspondence introduces a new approach to characterize textures at multiple scales. The performance of wavelet packet spaces are measured in terms of sensitivity and selectivity for the classification of twenty-five natural textures. Both energy and entropy metrics were computed for each wavelet packet and incorporated into distinct scale space representations, where each wavelet packet (channel) reflected a specific scale and orientation sensitivity. Wavelet packet representations for twenty-five natural textures were classified without error by a simple two-layer network classifier. An analyzing function of large regularity $\left(D_{20}\right)$ was shown to be slightly more efficient in representation and discrimination than a similar function with fewer vanishing moments $\left(D_{6}\right)$. In addition, energy representations computed from the standard wavelet decomposition alone (17 features) provided classification without error for the twenty-five textures included in our study. The reliability exhibited by texture signatures based on wavelet packets analysis suggest that the multiresolution properties of such transforms are beneficial for accomplishing segmentation, classification and subtle discrimination of texture.
\end{abstract}

Index Terms - Feature extraction, texture analysis, texture classification, wavelet transform, wavelet packet, neural networks.

\section{INTRODUCTION}

Texture is an important characteristic for the analysis of many types of images including natural scenes, remotely sensed data and biomedical modalities. The perception of texture is believed to play an important role in the human visual system for recognition and interpretation. Despite the lack of a complete and formal definition of texture, a large number of approaches for texture classification have been suggested [1]-[7].

Previous methods of analysis for accomplishing texture classification maybe roughly divided into three categories : statistical, structural and spectral. Statistical features based on second-order gray level statistics and gray level difference statistics have been studied extensively since the early 1970 s. R. Haralick et al. [10] proposed a set of 14 textural features extracted from a coocurrence matrix. They reported an overall accuracy rate of 84 percent on 11 types of textures obtained from satellite images. J. S. Weszka et al. [11] performed a comparative study on textural features. They argued that features based on second-order and difference statistics performed better than features obtained from a 2-D Fourier power spectrum They reported a 90 percent accuracy rate on 3 types of sample terrain images. More recently, model-based approaches related to random field models, have been investigated. Kashyap et al. [12] suggested a set of features based on a circular symmetric autoregressive random field model. They showed an average of 91 percent accuracy on the classification of 12 distinct types of natural textures. In addition,

Manuscript received July 24, 1992; revised March 15, 1993. This work was supported by the National Science Foundation under Grant IRI-9111375. Recommended for acceptance by Editor-in-Chief A. K. Jain.

The authors are with the Center for Computer Vision and Visualization, Computer and Information Sciences Department, Computer Science and Engineering Building, Room 301, University of Florida, Gainesville, FL. 32611-2024.

IEEE Log Number 9212240.
F. S.Cohen et al. [13] reported an 99-100 percent accuracy rate for nine types of natural textures using a Gaussian Markov random field model.

Although statistical features have been successful, psychophysicalexperiments have shown that features based on global second-order statistics are not complete. For example, B.Julesz et al. [14] and A. Gagalowicz [19] showed that some textures with the same global second-order statistics remain easily discriminable. This suggests that local second-order statistics may differ from global ones.

Experimental evidence on human and mammalian vision support the notion of spatial-frequency (multiscale) analysis that maximizes the simultaneous localization of energy in both spatial and frequency domains [15]-[18]. These recent findings have motivated several important studies. M. R.Turner [20] has applied Gabor functions to accomplish texture discrimination. A. C. Bovik et al. [21][22][23] and A. K. Jian et al. [24] and B. S. Manjunath et al. [26] used Gabor functions for the segmentation of texture. T. R.Reed et al. [27] demonstrated an approach to texture segmentation by using several spatial-frequency representations. J. R. Bergen and M. S. Landy [28] computed a pyramid structure of different spatial resolution and four orientation specific channels for visual texture segregation. M. Unser [8], [9] introduced local linear transforms for texture segmentation and classification. In this correspondence, we present a novel method of texture classification by multiresolution representations obtained from wavelet packet analysis.

Wavelet theory provides a precise and unified framework forspatialscale analysis. Carter [29] first reported texture classification results using Morlet and Mexican hat wavelets. He achieved 98 percent accuracy on 6 types of natural textures. However, these wavelets were not orthonormal, and the Mexican hat wavelet lacked direction selectivity. In this correspondence, these drawbacks are overcome by using orthonormal and compactly supported wavelets. The advantages are twofold. First, since the representation features at each scale are obtained by decomposing a signal (image) onto an orthonormal basis [30], correlation between scales is avoided. Second, orientation selectivity is built into the two-dimensional orthonormal wavelets included in our study. Experimentally, these capabilities provided outstanding sensitivity and selectivity for reliable discrimination. In this paper, we introduce a methodology for identifying texture representations based on wavelet packet analysis [31], [32]. We suggest that such analyses can provide a powerful method for accomplishing robust texture classification, compared to traditional single resolution techniques. The efficacy of the technique was rigorously demonstrated by the perfect classification of 1600 samples (no errors), obtained from 25 classes of natural textures.

In the next section, we review the wavelet packet decomposition and define thetwo measures of information compared as signatures for texture discrimination. Section III presents an overview of our strategy and experimental design. Methods of sample acquisition, selection of wavelet packet bases, and the performance of two classifiers are described. In Section IV, we present our results and discuss the performance with respect to the several parameters considered in our investigation. Finally, Section V summarizes the results and conclusions of our study. 


\section{WAVELET PACKET SIGNATURES}

Wavelet packets are a generalization of orthonormal and compactly supported wavelets [34], [30]. Pioneered by R. Coifman et al.[31], [32], methods of wavelet packets analysis have been successfully used for data compression [33]. Wavelet packets may be described by the collection of functions $\left\{W_{j}(x) \mid j \in Z^{+}\right\}$obtained from

$$
\begin{aligned}
& 2^{\frac{p-1}{2}} W_{2 n}\left(2^{p-1} x-l\right)=\sum_{m} h_{m-2 l} 2^{\frac{p}{2}} W_{n}\left(2^{p} x-m\right) \\
& 2^{\frac{p-1}{2}} W_{2 n+1}\left(2^{p-1} x-l\right)=\sum_{m} g_{m-2 l} 2^{\frac{p}{2}} W_{n}\left(2^{p} x-m\right)
\end{aligned}
$$

where $p$ is a scale index, $l$ is a translation index, $W_{0}(x)=\phi(x)$, $W_{1}(x)=\psi(x), \phi(x)$ is a scaling function and $\psi(x)$ is a basic wavelet [34], [30]. The discrete filters $h_{k}$ and $g_{k}$ are quadrature mirror filters [35], [34], [30].

We can show [31] that such wavelet packets are orthonormal in $L^{2}(R)$ and serve as bases similar to sinusoid functions in Fourier analysis. Furthermore, wavelet packets are well localized in both time and frequency and thus provide an attractive alternative to pure frequency (Fourier) analysis. The inverse relationship between wavelet packets of different scales can be shown by,

$$
\begin{aligned}
& 2^{\frac{p}{2}} W_{n}\left(2^{p} x-k\right)=\sum_{l} h_{k-2 l} 2^{\frac{p-1}{2}} W_{2 n}\left(2^{p-1} x-l\right) \\
&+\sum_{l} g_{k-2 l} 2^{\frac{p-1}{2}} W_{2 n+1}\left(2^{p-1} x-l\right) .
\end{aligned}
$$

Analogous to Fourier methods, any function $f(x) \in L^{2}(R)$ can be decomposed onto a wavelet packet basis. The coefficients of this decomposition are simply the inner products of $f(x)$ with distinct wavelet packets. For example, coefficients from the inner product $\left\langle f(x), W_{n}\left(2^{p} x-k\right)\right\rangle$ indicate the intensity of this component in $f(x)$. An approximation of an original function $f(x)$ using wavelet packet $W_{n}$ at scale $2^{p}$ can be written as

$$
A_{n}^{2^{p}} f(x)=\sum_{k} S_{n, k}^{p} 2^{\frac{F}{2}} W_{n}\left(2^{p} \cdot x-k\right)
$$

where

$$
S_{n, k}^{p}=2^{\frac{p}{2}} \int_{-\infty}^{\infty} f(x) W_{n}\left(2^{p} x-k\right) d x .
$$

Next, we show how wavelet packets may be computed efficiently. From (3), we may write

$$
S_{n, k}^{p}=\sum_{l} h_{k-2 l} S_{2 n, l}^{p-1}+\sum_{l} g_{k-2 l} S_{2 n+1, l}^{p-1} .
$$

Using (1) and (2), coefficients at coarser scales are calculated by

$$
\begin{aligned}
S_{2 n, l}^{p-1} & =\sum_{m} h_{m-2 l} S_{n, m}^{p} \\
S_{2 n+1, l}^{p-1} & =\sum_{m} g_{m-2 l} S_{n, m}^{p} .
\end{aligned}
$$

Note that for standard wavelet decompositions [30], only two wavelet packets $W_{0}$ and $W_{1}$ are used. In this case, the index $n$ is restricted to $n=0$, and only nodes $S_{0}^{p}$ are decomposed from (7) and (8). Thus only the leftmost node at each level has children, and each level has exactly two nodes. Thus from the subband filtering (filter bank) point of view, the difference between a wavelet packet decomposition and the standard wavelet transform is that the former recursively decomposes the high-frequency components, thus constructing a tree-structured multiband extension of the wavelet transform.

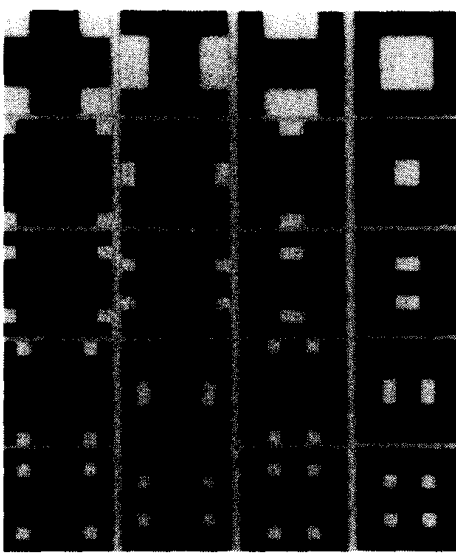

Fig. 1. Frequency responses of discrete filters, where $(i, j)$ denotes rowi, colomn $j .(1,1)$ : level 1 , diagonal channel; $(1,2)$ : level 1 ,vertical channel; $(1,3)$ : level 1 , horizontal channel; $(1,4)$ : level 1 , dc channel. $(2-5,1)$ : level 2 , four children of $(1,1) ;(2-5,2)$ : level 2 , four children of $(1,2) ;(2-5,3)$ : level 2 , four children of $(1,3) ;(2-5,4)$ : level 2 , four children of $(1,4)$

For discrete signals, we treat the original discrete signal as the set of wavelet packet coefficients at the first scale $(p=0)$, and then apply the technique previously described. Since we process digitized samples of textures, we focus on discrete signals for the rest of the correspondence.

The basis functions are obtained by translation and scale change. They remain well localized in both time (spatial) and frequency domains and thus represent scale and spatial information. Thus, a complete tree presents the distribution of a signal within a scale space continuum. Note that the total number of coefficients in a complete tree decomposition is exactly equal to the number of points (pixels) in an original signal. Energy distributions within transform spaces have been applied in Fourier analysis [3]. Similarly, we will show similar significance in wavelet packet analysis. Since wavelet packets form orthogonal bases, their decompositions will preserve energy. It is easy to show that

$$
\sum_{k}\left(S_{n, k}^{p}\right)^{2}=\sum_{l}\left(S_{2 n, l}^{p-1}\right)^{2}+\sum_{l}\left(S_{2 n+1, l}^{p-1}\right)^{2} .
$$

Therefore, if we define a energy measure as $E_{n}^{p}=\sum_{k}\left(S_{n, k}^{p}\right)^{2}$, then

$$
E_{n}^{p}=E_{2 n}^{p-1}+E_{2 n+1}^{p-1} .
$$

Our strategy was to first compute the energy associated within each wavelet packet. We hypothesized that the energy pattern distributed in scale space shall provide unique information, and support a representation (signature) for classification. Thus, a signature was a feature vector consisting of a set of energy values. In this correspondence, we demonstrate that such signatures can provide a powerful and efficient means to accomplish signal classification.

An alternative measure of information is entropy, defined by,

$$
H(x)=-\sum_{k}\left|x_{k}\right|^{2} \log \left|x_{k}\right|^{2} .
$$

This measure was previously proposed in [10] for texture analysis, and has also been used in [36] to identify a "best basis" for building wavelet packet libraries for signal compression. In this paper, we compare the entropy and energy measures described above for their performance in accomplishing robust texture discrimination.

The extension for 2-D signals is straight forward by using aspecial class of separable 2-D wavelet packets. In this case, the energy 


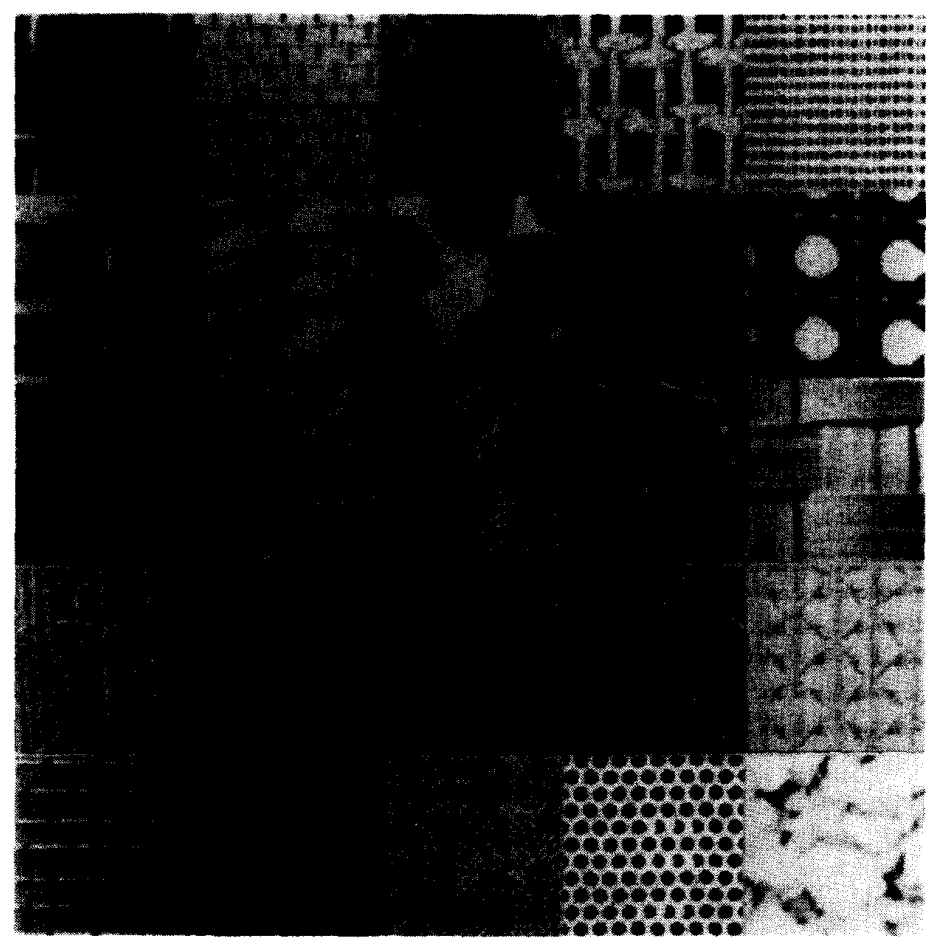

Fig. 2. Twenty-five natural textures.row $1: D 1 . D 6 . D 11 . D 20 . D 21:$ row $2: D 56 . D 65 . D 75 . D 82 . D 101$ row 3 : D55. D84. D81. D110. D64: row 4: Dis. D17. D5. D4. D52: row 5: D14. D8, D16. O1. O2.

preserving equation is specified by the sum

$$
E_{n, m}^{p}=E_{2 n, 2 m}^{p-1}+E_{2 n \cdot 2 m+1}^{p-1}+E_{2 n+1.2 m}^{p-1}+E_{2 n+1,2 m+1}^{p-1} .
$$

Orientation selectivity is embedded in the tensor product of the lowpass filter $h$ and high-pass filter $g$, and therefore, energy distributions are captured in three orientations (subbands). Fig. 1 shows the set of equivalent frequency channels associated with a wavelet packet transform for levels one and two.

\section{METHODOLOGY}

In this section, we present our experimental design and analytic methods for accomplishing texture classification using wavelet packet representations.

\section{A. Texture Selection and Sampling}

Twenty-three distinct natural textures were selected from the Brodatz album [37] and two additional textures were obtained from public archive. The complete set and diversity of the twenty-five texture classes are shown in Fig. 2. Each texture class was digitized on a Microtek $300 \mathrm{Z}$ scanner at 130 samples/in density on a Sun $4 / 75$ workstation, and stored as a $j 12 \times j 12,8 \mathrm{bit} / \mathrm{pixel}$ digital image. Each texture class was then broken down into $128 \times 128$ sub-samples. Our selection criteria was such that each texture pattern sample maintained a certain degree of spatial periodicity within its $128 \times 128$ matrix size.

Both a neural network and a minimum-distance classifier were usedto accomplish supervised classification. To obtain a large amount of data for training the classifiers, we adapted a method of overlapped sampling. We extracted 64 sub-samples of size $128 \times 128$ (pixels) from each original $512 \times 512$ sample texture. The amount of overlap was fixed at 43 pixels for extracting all sub-samples.

\section{B. Partitions of Wavelet Packet Space}

A complete set of wavelet packets were computed for each $128 \mathrm{x}$ 128 subsample. The discrete filters $D_{6}$ and $D_{20}$ were obtained from Daubechies [34]. Due to downsampling at each decomposition step, the size of each subsample was reduced by a factor of four. Thus, a subsample at level four, consisted of $64(8 \times 8)$ coefficients. Recall that each "parent" node has four "children." Therefore, a complete five-level decomposition (levels 0 through 4) consisted of exactly 341 wavelet packets.

We measured the energy (and entropy) contributed by each wavelet packet,and treated its real value as a distinct feature element in a vector. Thus, in our representation, the maximum number of features encoded for a sample texture consisted of a vector of 341 real values. However, we investigated the classification performance of each signature supported by distinct subsets of wavelet packet nodes. Subset were defined by complete and overcomplete (redundant) representations. We considered six distinct partitions of wavelet packets selected from a complete decomposition tree (full recursion) and measured their performance in terms of sensitivity and selectivity for the discrimination of all twenty-five classes of natural texures. Next, we identify the six partitions of wavelet packet nodes that provided the bases for feature representation in our study.

1) Complete set of wavelet packet nodes: Each texture sub-sample was represented by a vector of 341 features.

2) Standard wavelets: As mentioned earlier, nodes of the standard wavelet decomposition (Mallat [30]) are a subset of a complete wavelet packet decomposition. In this case, the four leftmost nodes at each level of the complete wavelet packet decomposition tree were selected. Thus, cach sub-sample was represented by only 17 features. 


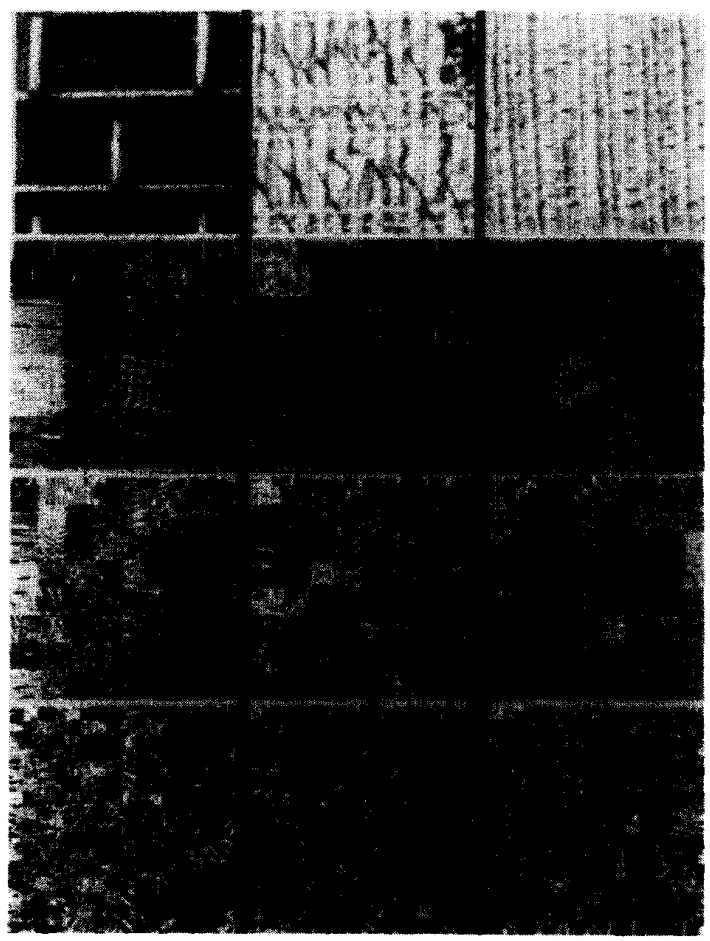

Fig. 3. Three sample textures ( row $\left._{1}\right)$, and magnitude of wavelet packet coefficients for decomposition levels 2,3 , and 4 ( row $_{2}$. row 3 . row row $_{4}$.

3) Levels 1, 2 and 3: In this case, each texture sample was represented by exactly 84 features. Energy features computed from wavelet packet nodes of levels 0 and 4 were discarded.

4) Levels 2 and 3: Each texture sample was represently by exactly 80 features.

5) Level 3: Each texture sample was represented by exactly 64 features.

6) Level 4: Each texture sample was represented by exactly 256 features. In this case, energy features contributed from a single coarse scale.

For each of the six representations, we computed feature vectors for the 64 subsamples of each texture class. Our database consisted of 42 samples for classification training and 22 samples for testing. Therefore, in total, our study processed $1050(42 \times 25)$ sample signatures in training and $550(22 \times 25)$ sample signatures in testing classification performance.

In Fig. 3, we show three samples of texture, and themagnitude of their wavelet packet coefficients for levels 2,3 , and 4 (from top to bottom). Fig. 4 displays in pseudo-color, energy signatures corresponding to the wavelet packet coefficients pictured in Fig. 3 . The color map was obtained by first computing the logarithm for each feature value, then globally scaling the values within a 0-255 range. Therefore, Fig. 4 shows a normalized energy distribution for each signature.

\section{Discrimination Using a Simple Minimum-Distance Classifier}

To decide the efficacy of wavelet packet signatures for texture classification, the performance of a simple minimum-distance classifier was evaluated. A single prototype minimum-distance classifier [40] was based on the assumption that each pattern class $\omega_{k}$ is representable by a prototype pattern $\mathbf{Z}_{k}$ (class center). The minimumdistance classifier assigned a pattern $\mathbf{X}$ of unknown classification to

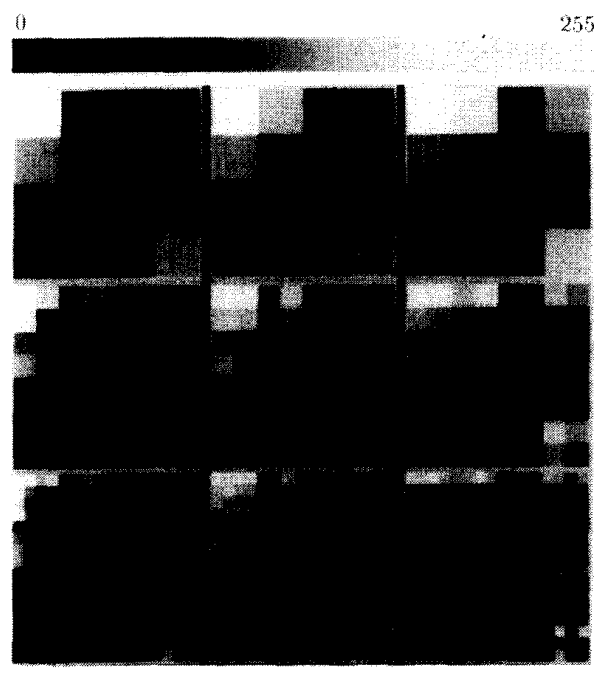

Fig. 4. Log energy maps for wavelet packet nodes of levels 2, 3, and 4, for the texture samples shown in Fig. 3.

the class $\alpha^{\prime} \mathbf{k}$, if the distance $D_{k}$ between $\mathbf{X}$ and $\mathbf{Z}_{k}$ was minimum among all possible class prototypes $\omega_{j} \neq \omega_{k}$, where the Euclidean distance $D_{k}$ was defined by

$$
D_{k}=\left\|\mathbf{X}-\mathbf{Z}_{k}\right\|=\sqrt{\sum_{\mathbf{j}=\mathbf{0}}^{\mathbf{N}-\mathbf{1}}\left(x_{j}-z_{k, j}\right)^{2}} .
$$

In practice, the true class centers $\mathbf{Z}_{k}$ 's are also unknown. In our case (supervised classification), each class center $\mathbf{Z}_{k}$ was estimated by using the mean of the training sample for each class $\omega_{k}$,

$$
\mathbf{Z}_{k}=\frac{1}{\gamma_{k}} \sum_{\mathbf{X} \in \omega_{k}} \mathbf{X} .
$$

The estimated class center minimized a performance index defined by

$$
\mathbf{J}_{k}=\sum_{\mathbf{X} \in \omega_{k}}\left\|\mathbf{X}-\mathbf{Z}_{k}\right\|^{2} \cdot k=1,2, \cdots, M .
$$

where $I I$ was the total number of texture classes (twenty-five). To discriminate the subtle differences between some of the texture classes included in our study, we engaged a more sophisticated classifier, described in the next section.

\section{Discrimination Using a Neural Network Classifier}

We examined the classification performance of each wavelet packet representation for several network topologies. We used a two-layer back propagation network [38] having a conjugate gradient function for error correction [39]. For each topology, the number of input nodes was matched to the vector dimension (number of real values) comprising each wavelet packet representation. All network topologies had twenty-five output nodes, the total number of distinct texture classes.

By using wavelet packet representations, we reduced the number of bits required for each original texture pattern by a factor of 240 $\left(\frac{128 \times 128 \times 8}{17 \times 32}\right)$. Thus we were able to reduce the number of input nodes (bandwidth) of a neural network by a factor of $960\left(\frac{128 \times 128}{17}\right)$.

\section{RESULTS AND DISCUSSION}

In this section, we summarize the discrimination results obtained for the twenty-five classes of natural textures. In Table I, we show 
TABLE 1

Classification Results Comparing the Performance of Two Signaturemetrics, Complete and OVERCOMPlete WaVelet PaCket Representations and Two analyzing Functions

\begin{tabular}{|c|c|c|c|c|c|}
\hline Sig. & $\begin{array}{l}\text { Selected } \\
\text { Wavelet } \\
\text { Packets }\end{array}$ & $N$ & $\begin{array}{l}\text { Analy. } \\
\text { Func. }\end{array}$ & $\begin{array}{c}\text { Num. } \\
\text { of } \\
\text { Err. }\end{array}$ & $\begin{array}{c}\% \% \\
\text { Correct }\end{array}$ \\
\hline \multirow{4}{*}{ E } & \multirow[t]{2}{*}{ Comp. } & \multirow[t]{2}{*}{341} & $D_{20}$ & 0 & 100 \\
\hline & & & $D_{6}$ & 0 & 100 \\
\hline & \multirow[t]{2}{*}{ Stand. } & \multirow[t]{2}{*}{17} & $D_{20}$ & 0 & 100 \\
\hline & & & $D_{6}$ & 4 & 99.3 \\
\hline \multirow{4}{*}{ H } & \multirow[t]{2}{*}{ Comp. } & \multirow[t]{2}{*}{341} & $\overline{D_{20}}$ & $\overline{0}$ & 100 \\
\hline & & & $D_{B}$ & 0 & 100 \\
\hline & \multirow[t]{2}{*}{ Stand. } & \multirow[t]{2}{*}{17} & $D_{20}$ & 1 & 99.8 \\
\hline & & & $D_{6}$ & 5 & 99.1 \\
\hline
\end{tabular}

E: energy, H: entropy, $N$ : number of features.

TABLE II

Classification Results for Energy Signatures Computed from Redundant and Complete WaVelet Packet Representations, Using a $D_{20}$ ANALYZING Function

\begin{tabular}{c|c|c|c}
\hline $\begin{array}{c}\text { Selected } \\
\text { Wavelet } \\
\text { Packets }\end{array}$ & $\begin{array}{c}\text { Number } \\
\text { of } \\
\text { Features }\end{array}$ & $\begin{array}{c}\text { Number } \\
\text { of } \\
\text { Errors }\end{array}$ & \% Correct \\
\hline \hline Level $1,2,3$ & 84 & 1 & 99.8 \\
\hline Level 2,3 & 80 & 0 & 100 \\
\hline Level 3 only & 64 & 0 & 100 \\
\hline Level 4 only & 256 & 0 & 100 \\
\hline
\end{tabular}

the performance of three parameters included in our study: a) Energy versus entropy based signature metrics, b) a minimal (standard) and maximum (overcomplete) number of wavelet packet nodes for representation, c) $D_{20}$ (long FIR) versus $D_{6}$ (short FIR) analyzing functions. When using overcomplete (redundant) wavelet packet representations, perfect classification for twenty-five textures was observed regardless of the signature (energy versus entropy) or analyzing wavelet ( $D_{6}$ versus $D_{20}$ ) included. However, when texture signatures were computed from a minimal (complete) number of wavelet packet nodes (i.e., the standard wavelet tree), perfect classification was observed only for the $D_{20}$ analyzing wavelet. For sensitive applications, this demonstrates that a) longer (FIR) analyzing functions are more efficient for discriminating salient textural features b) perfect classification is achievable by a minimal representation of energy, based on 17 wavelet packet nodes of a four level decomposition. For the textures included in our investigation, we observed that signatures computed from energy performed only slightly better than entropy based representations.

In Table II, we show the classification performance of energy signatures computed from redundant and complete sets of wavelet packet representations. Signatures computed from wavelet packets of level 3 alone ( 64 features) and level 4 alone (256 features) yielded perfect classification results for all twenty-five texture classes. However, texture signatures computed from levels 1, 2, and 3 (redundant representations) resulted in a classification error! This suggests that redundancy may increase uncertainty (degrees of freedom) for the classifier employed in our study.

Table III compares the performance of network topologies for the two analyzing functions $\left(D_{6}\right.$ and $\left.D_{20}\right)$. Energy signatures for samples of the twenty-five texture classes were computed from a standard wavelet decomposition (17 wavelet packets) and trained for classification. Classification results for five topologies are reported, where the number of hidden nodes varied from one to five in each case. The neural network classifier was trained and tested independently for each configuration using the same data set. In the case of the $D_{6}$ analyzing function, errors were observed for all five network topologies, Perfect classification was observed only for
TABLE III

Network Training Times and Topologies for Classification Using $D_{6}$ and $D_{20}$ Analyzing Functions

\begin{tabular}{c|c|c|c|c|c}
\hline \hline & Hidd. & Training & $\begin{array}{c}\text { Train. } \\
\text { Time } \\
\text { Nodes }\end{array}$ & $\begin{array}{c}\text { Num. } \\
\text { of } \\
\text { Enr. }\end{array}$ & $\begin{array}{c}\% \\
\text { Correct }\end{array}$ \\
\hline \multirow{3}{*}{$D_{6}$} & 1 & 225 & 50740 & 1 & 99.8 \\
\cline { 2 - 6 } & 2 & 226 & 53770 & 3 & 99.5 \\
\cline { 2 - 6 } & 3 & 102 & 24828 & 4 & 99.3 \\
\cline { 2 - 6 } & 5 & 32 & 8300 & 8 & 98.5 \\
\hline \hline \multirow{5}{*}{$D_{20}$} & 1 & 153 & 35231 & 2 & 99.6 \\
\cline { 2 - 6 } & 2 & 125 & 29857 & 1 & 99.8 \\
\cline { 2 - 6 } & 3 & 21 & 5203 & 0 & 100 \\
\cline { 2 - 6 } & 5 & 33 & 8822 & 1 & 99.8 \\
\hline
\end{tabular}

the $D_{20}$ analyzing function when the network consisted of exactly three hidden nodes. Note that fewest training epochs were required in this case. In general, signatures computed from $D_{6}$ analyzing functions required significantly more training time than signatures obtained from $D_{20}$ analyzing functions. In particular, we observed an almost five-fold difference in the number of epochs required to train a network classifier containing three hidden nodes (21 epochs for $D_{20}$, versus 102 epochs for $D_{6}$ ). This suggests that longer (FIR) analyzing functions (high regularity) may provide a more efficient representation for texture discrimination.

Even the simple minimum-distance classifier using wavelet packet signatures from level 3 alone was able to discriminate 550 sample patterns $(22$ samples/texture $\times 25$ textures) with 96 percent (not shown above) accuracy. This result supports our claim that texture signatures computed from wavelet packet energies alone, can be highly efficient representations for texture analysis.

In the next section, we summarize the results of our investigation.

\section{SUMMARY AND CONCLUSION}

Wavelet packet representations for twenty-five natural textures were classified without error by a simple two-layer network classifier. A longer analyzing function was shown to be more efficient in representation and discrimination than a similar function of shorter length. Experimentally, we observed that a neural network classifier performed best in terms of accuracy and minimal training time when configured with exactly three hidden units.

Energy representations computed from the standard set of wavelet nodes alone were sufficient for errorless classification. However, finer discrimination may be more strongly supported by additional subsets of wavelet packets (redundancy). We suggest that identifying an "optimal" set of wavelet packets for texture representation may depend on the aggregate of textural features targeted for classification. Thus, similar textures consisting of variations mostly at finer scales may be best discriminated by representations computed from wavelet packets of higher levels.

With respect to the $128 \times 128$ pixels/sample sizes included in this study, we observed that representations computed from level three (64 feature values) exhibited acute selectivity and sensitivity typically required for autonomous texture analysis. Texture signatures computed from wavelet packet energy performed only slightly better than entropy signatures computed from the same wavelet packets. In this study, analyzing functions were constructed by computing the tensor product of one-dimensional functions, and exhibited sensitivity along the principal axes. Finer discrimination may be possible by using two-dimensional nonseparable analyzing functions such as hexagonal wavelets having more orientation selectivity.

Texture signatures based on multiresolution wavelet packet analysis holds a great potential for accomplishing robust classification and subtle discrimination. In addition, methods of scale-space analysis 
for texture segmentation may benefit by the techniques and efficient representations suggested by these results.

\section{REFERENCES}

[1] R. M. Haralick, "Statistical and structural approaches to texture," Proc IEEE, vol. 67, pp. 786-804, 1979.

[2] L. S. Davis, "Image texture analysis techniques survey," in Digita Image Processing, J. C. Simon and R. M. Haralick, Eds. Dordrecht, The Netherlands: D. Reidel, 1980 , pp. 189-201.

[3] R. Bajcsy, "Computer description of textured surfaces," in Proc. 3rd Int Joint Conf. Artificial Intell., Aug. 1973, 572-579.

[4] C. H. Chen, "A study of texture classification using spectral features," in Proc. IEEE 6th Int. Conf. Pattern Recongnit., Munich, Germany, Oct. 19-22, 1982, pp. 1074-1077

[5] V. L. Vickers and J. W. Modestino, "A maximum likelihood approach to texture classification," IEEE Trans. Pattern Anal. Machine Intell., vol. PAMI-4, pp. 61-68, 1982.

[6] R. Wilson and M. Spann, Image segmentation and uncertainty. London: Research Studies Press Ltd, 1988

[7] M. Unser, "Sum and difference histograms for texture classification,"IEEE Trans. Pattern Anal. Machine Intell., vol. PAMI-8, pp. 118-125, 1986.

[8] __ Local linear transforms for texture measurements," Acoustics, Speech, Signal Processing, vol. ASSP-11, pp. 61-79, July 1986.

[9] M. Unser and M. Eden, "Multiresolution feature extraction and selection for texture segmentation," IEEE Trans. Pattern Anal. Machine Intell. vol. 11, pp. 717- 728, 1989.

[10] R. M. Haralick, K. Shanmugam, and I. Dinstein, "Textural features for image classification," IEEE Trans. Syst., Man, Cybern., vol. SMC-3, pp 610-621, 1973.

[11] J. S. Weszka, C. R. Dyer, and A. Rosenfield, "Comparative study of texture measures for terrain classification," IEEE Trans. Syst., Man, Cybern., vol. SMC-6, pp. 269-285, 1976.

[12] R. L. Kashyap and A. Khotanzad, "A model-based method for rotation invariant texture classification," IEEE Trans. Pattern Anal. Machine Intell, vol. PAMI-8, pp. 472-481, 1986.

[13] F. S. Cohen, Z. Fan, and M. A. Patel, "Classification of rotated and scaled textured images using Gaussian Markov random field models," IEEE Trans. Pattern Anal. Machine Intell., vol. 13, pp. 192-202, 1991.

[14] B. Julesz, E. N. Gilbert, J. D. Victor, "Visual discrimination of textures with identical third-order statistics," Biol. Cybern., vol. 31, pp. 137-140, 1978

[15] B. Julesz, "A theory of preattentive texture discrimination based on first-order statistics of textons," Biol. Cybern., vol. 41, pp. 131-138, 1981.

[16] R. De Valois, and K. De Valois, Spatial Vision. New York: Oxford Univ. Press, 1988.

[17] S. Marcelja, "Mathematical description of the responses of simple cortia cells," J. Opt. Soc. Am., vol. 70, pp. 1297-1300, Nov. 1980.

[18] J. Beck, A. Sutter, and R. Ivry, "Spatial frequency channels and perceptual grouping in texture segregation," Comput. Vision, Graphics, Image Processing, vol. 37, pp. 299-325, 1987.

[19] A. Gagalowicz, "A new method for texture fields synthesis: Some applications to the study of human vision," IEEE Trans. Pattern Anal. Machine Intell., vol. PAMI-3, pp. 520-533, 1982.

[20] M. R. Turner, "Texture discrimination by Gabor functions," Biol. Cybern., vol. 55, pp. 71-82, 1986.

[21] A. C. Bovik and M. Clark, "Multichannel texture analysis using localized spatial filters," IEEE Trans. Pattern Anal. Machine Intell., vol. 12 pp. 55-73, 1990.

[22] A. C. Bovik, "Analysis of multichannel narrowband filters for image texture segmentation," IEEE Trans. Signal Processing, vol. 39, pp 2025-2043, Sept. 1991.

[23] A. C. Bovik, N. Gopal, T. Emmoth, and A. Pestrepo, "Localized measurement of emergent image frequencies by Gabor wavelets," IEEE Trans. Inform. Theory, vol. 38, pp. 691-712, Mar. 1992.

[24] A. K. Jain and F. Farrokhnia, "Unsupervised texture segmentation using Gabor filters," Pattern Recognit., vol. 24, no. 12, pp. 1167-1186, 1991.

[25] A. K. Jain and S. Bhattacharjee, "Text segmentation using Gabor filters for automatic document processing," Machine Vision and Applicat., vol. 5, pp. $169-184,1992$
[26] B. S. Manjunath and R. Chellappa, "A computational approach to boundary detection," Proc. CVPR, 1991, pp. 358-363.

[27] T. R. Reed and H. Wechsler, "Segmentation of textured images and gestalt organization using spatial/spatial-frequency representations," IEEE Trans. Pattern Anal. Machine Intell., vol. 12, pp. 1-12, Jan. 1990.

[28] J. R. Bergen and M. S. Landy, "Computational modeling of visual texture segregation," in Computational Models of Visual Processing, M. S. Landy and J. A. Movshon, Eds. Cambridge, MA: The MIT Press, 1991, pp. 472-481.

[29] P. H. Carter, "Texture discrimination using wavelets," in SPIE applica tions of digital image processing XIV, vol. 1567, pp. 432-438, 1991.

[30] S. Mallat, "A theory for multiresolution signal decomposition: The wavelet representation," IEEE Trans. Pattern Anal. Machine Intell., vol. 11 , pp. $674-693,1989$.

[31] R. R. Coifman and Y. Meyer, "Orthonormal wave packet bases," preprint, Yale Univ., Aug., 1989.

[32] R. R. Coifman and M. V. Wickerhauser, "Best-adapted wavelet packet bases," preprint, Yale Univ., Feb. 1990

[33] _ "Entropy-based algorithms for best basis selection," IEEE Trans. Inform. Theory, vol. 38, pp. 713-718, May 1992.

[34] I. Daubechies, "Orthonormal bases of compactly supported wavelets," Commun. Pure Appl. Math., vol. XLI, pp. 909-996, 1988.

[35] R. E. Crochiere and L. R. Rabiner, Multirate digital signal processing. Englewood Cliffs, NJ: Prentice-Hall, 1983.

[36] R. R. Coifman et al., "Signal processing and compression with wave packets," preprint, Yale Univ.

[37] P. Brodatz, Textures - A Photographic Album for Artists and Designers. New York: Dover, 1966.

[38] D. E. Rumelhart and J. L. McClelland, Parallel Distributed Processing. Cambridge, MA: MIT Press, 1986.

[39] B. L. Kalman, "Super linear learning in back propagation neural nets," Wucs-90-21, Washington Univ., St. Louis, MO, 1990.

[40] J. T. Tou and R. C. Gonzalez, Pattern Recognition Principles. Reading, MA: Addison-Wesley, 1974; second printing 1977.

\section{B-Spline Contour Representation and Symmetry Detection}

Philippe Saint-Marc, Hillel Rom, and Gérard Medioni

Abstract- The detection of edges is only one of many steps in the understanding of images. Further processing necessarily involves grouping operations between contours. We present a representation of edge contours by approximating B-splines and show that such a representation facilitates the extraction of symmetries between contours. Our representation is rich, compact, stable, and does not critically depend on feature extraction. We turn our attention to the detection of three types of symmetries: skew symmetries and parallel symmetries, which have proven to be of great importance in inferring shape from contour, and smooth local symmetries, which have been used for planar shape description. We show that our representation facilitates the computation of these symmetries.

Index Terms-B-splines, contour representation, shape representation, spline approximation, symmetry, symmetry detection.

Maunscript received September 6, 1991; revised July 13, 1992. This work was supported by the Advanced Research Projects Agency of the Department of Defense and was monitored by the Air Force Office of Scientific Research under Contract F49 620-90-C-0078. The United States Government is authorized to reproduce and distribute reprints for governmental purposes notwithstanding any copyright notation hereon. A considerably shorter version of this paper has been published in ECCV-90 (see [38]). Recommended for acceptance by Associate Editor T. Boult.

P. Saint-Marc is with Matra-MS2I, Signal and Image Processing Laboratory, St-Quentin en Yvelynes Cédex, France.

H. Rom and G. Medioni are with the Institute for Robotics and Intelligent Systems, University of Southern California, Los Angeles, CA 90089-0273.

IEEE Log Number 9211833. 